\title{
ON CERTAIN COEFFICIENTS OF UNIVALENT FUNCTIONS. II $\left({ }^{1}\right)$
}

BY

\author{
JAMES A. JENKINS
}

1. In the first paper with this title [4] the author showed how an extension of the General Coefficient Theorem in the case of some special quadratic differentials gave a very effective means of dealing with bounds for initial coefficients of normalized univalent functions. Since that time the author has given a corresponding extension of the General Coefficient Theorem to the most general positive quadratic differentials on finite oriented Riemann surfaces [5]. In the present paper we will apply this result to the discussion of the coefficients of normalized univalent functions. Some authors, see for example [6], have discussed the effect on the coefficients of a univalent function of having certain initial coefficients vanish while others $[1 ; 2]$ have obtained bounds for the coefficients of univalent functions which are odd or display higher degrees of symmetry. We will find a very interesting relationship between these two types of results.

2. We will consider the families $S$ and $\Sigma$ of functions according to the standard notation [3, p. 1] and in addition the following family of functions.

DEFINITION 1. Let $M$ denote the family of functions $f(z)$ meromorphic and univalent for $|z|<1$ with $f(0)=0, f^{\prime}(0)=1$.

We have the evident result.

Lemma 1. We have $f(z) \in \Sigma$ if and only if $1 / f(1 / z) \in M$.

We begin by giving certain mappings which play the role of extremal mappings.

Lemma 2. Let $Q(w ; n, \delta, \psi) d w^{2}$ be the quadratic differential

$$
e^{-2 n \psi i} w^{n-2}\left(\delta e^{n \psi i}-w^{n}\right) d w^{2}
$$

where $n$ is a positive integer, $\psi$ is real and $0 \leqq \delta \leqq 4$. For $0<\delta \leqq 4$ let $f(z ; n, \delta, \psi)$ be the function in $\Sigma$ mapping $|z|>1$ conformally onto the domain $\Delta(n, \delta, \psi)$ bounded by the $n$ rectilinear segments joining the origin to the points $\delta^{1 / n} e^{i(\psi+2 \pi j / n)}, j=0,1, \cdots, n-1$, together with $2 n$ arcs of equal length on the other trajectories of $Q(w ; n, \delta, \psi) d w^{2}$ having limiting end points at these zeros of the quadratic differential (when $\delta=4$ the last $2 n$ arcs are absent). Let $f(z ; n, 0, \psi)$

Received by the editors December 10, 1959.

(1) This work was sponsored by the Office of Ordnance Research, U. S. Army, Contract no. DA-36-034-ORD-2884 RD. 
be the function in $\Sigma$ mapping $|z|>1$ conformally onto the domain $\Delta(n, 0, \psi)$ bounded by $2 n$ rectilinear segments of equal length emerging from the origin along trajectories of $Q(w ; n, 0, \psi) d w^{2}$. We have

$$
f(z ; n, 0, \psi)=z\left(1-e^{2 n \psi i} z^{-2 n}\right)^{1 / n}
$$

the root being chosen so that the expansion of $f(z ; n, 0, \psi)$ about the point at infinity begins

$$
z-\frac{1}{n} e^{2 n \psi i} z^{-2 n+1}+\cdots
$$

The functions which can be obtained from $f(z ; n, 0, \psi), n \geqq 2$, by translation along the trajectories of $Q(w ; n, 0, \psi) d w^{2}$ are a one parameter family $F(z ; n, 0, \psi, \mu)$ where

$$
F(z ; n, 0, \psi, \mu)=z\left(1+i n \mu e^{n \psi i} z^{-n}-e^{2 n \psi i} z^{-2 n}\right)^{1 / n},
$$

the root is chosen so that the expansion of $F(z ; n, 0, \psi, \mu)$ about the point at infinity begins

$$
z+i \mu e^{n \psi i} z^{-n+1}+\left(\frac{n-1}{2} \mu^{2}-\frac{1}{n}\right) e^{2 n \psi i_{z}-2 n+1}+\cdots
$$

and $\mu(-2 / n \leqq \mu \leqq 2 / n)$ denotes the (sensed) amount of translation in the $Q$ metric. The same formulae remain valid for $n=1$ if we restrict ourselves to functions not assuming the value zero for $|z|>1$. For $0<\delta \leqq 4$ the expansion of $f(z ; n, \delta, \psi)$ about the point at infinity begins

$$
\begin{aligned}
z & +\frac{1}{2 n} \delta[1-\log (\delta / 4)] e^{n \psi i} z^{-n+1} \\
& +\left[\frac{1}{8 n^{2}} \delta^{2}-\frac{1}{4 n^{2}} \delta^{2} \log (\delta / 4)-\frac{(n-1)}{8 n^{2}} \delta^{2}[\log (\delta / 4)]^{2}-\frac{1}{n}\right] e^{2 n \psi i_{z}-2 n+1}+\cdots
\end{aligned}
$$

When $0<\delta<4$ there can be obtained from $f(z ; n, \delta, \psi)$ by translation along the trajectories of $Q(w ; n, \delta, \psi) d w^{2}$ a one parameter family of functions $F(z ; n, \delta, \psi, \mu)$ where $\mu$ denotes the (sensed) amount of translation and satisfies the inequalities

$$
-\frac{2}{n}\left(1-\frac{1}{16} \delta^{2}\right)^{1 / 2}+\frac{1}{2 n} \delta \cos ^{-1} \frac{\delta}{4} \leqq \mu \leqq \frac{2}{n}\left(1-\frac{1}{16} \delta^{2}\right)^{1 / 2}+\frac{1}{2 n} \delta \cos ^{-1} \frac{\delta}{4} \text {. }
$$

In particular

$$
F(z ; n, \delta, \psi, 0) \equiv f(z ; n, \delta, \psi) .
$$

The expansion of $F(z ; n, \delta, \psi, \mu)$ about the point at infinity begins 


$$
\begin{aligned}
z+ & \frac{1}{n}\left[n \mu i+\frac{1}{2} \delta(1-\log (\delta / 4))\right] e^{n \psi i} z^{-n+1} \\
+ & {\left[\frac{1}{2 n} \delta \mu i+\left(\frac{n-1}{2}\right) \mu^{2}+\left(\frac{n-1}{2 n}\right) i \mu \delta \log (\delta / 4)+\frac{1}{8 n^{2}} \delta^{2}-\frac{1}{4 n^{2}} \delta^{2} \log (\delta / 4)\right.} \\
& \left.-\frac{(n-1)}{8 n^{2}} \delta^{2}(\log (\delta / 4))^{2}-\frac{1}{n}\right] e^{2 n \psi i z^{-2 n+1}}+\cdots
\end{aligned}
$$

For $\delta=4$ there are no functions obtained from $f(z ; n, 4, \psi)$ by nontrivial translation along the trajectories of $Q(w ; n, 4, \psi) d w^{2}$.

For $\delta=0$ the result of the lemma is elementary. For $0<\delta \leqq 4$, in terms of the notation of $[4$, Lemma 1$]$, if we set $W=w^{n}, Z=z^{n}, \tau=\delta, \phi=n \psi, \lambda=n \mu$ we readily find

$$
\begin{aligned}
Q_{\phi}(W) d W^{2} & =n^{2} Q(w ; n, \delta, \psi) d w^{2}, \\
{[f(Z, \tau, \phi)]^{1 / n} } & =f(z ; n, \tau, \psi), \\
{[F(Z, \tau, \phi, \lambda)]^{1 / n} } & =F(z ; n, \tau, \psi, \mu)
\end{aligned}
$$

with appropriate choices of the roots. From this the remaining results of the lemma follow directly. It will be observed that our notation is somewhat modified from that of [4].

Lemma 3. Let $Q(w ; n, \chi) d w^{2}$ be the quadratic differential

$$
e^{-i n x_{w} w^{n-2}} d w^{2}
$$

where $n$ is a positive integer and $\chi$ is real. Let $g(z ; n, \chi)$ be the function in $\Sigma$ mapping $|z|>1$ conformally onto the domain $\Delta(n, \chi)$ bounded by $n$ rectilinear segments of equal length emanating from the origin along trajectories of $Q(w ; n, \chi) d w^{2}$. Then

$$
g(z ; n, \chi)=z\left(1+e^{n \times i_{i}} z^{-n}\right)^{2 / n}
$$

the root being chosen so that the expansion of $g(z ; n, \chi)$ about the point at infinity begins

$$
z+\frac{2}{n} e^{n x^{i} z^{-n+1}}+\cdots
$$

When $n$ is odd no functions can be obtained from $g(z ; n, \chi)$ by nontrivial translation along the trajectories of $Q(w ; n, \chi) d w^{2}$. When $n$ is even and greater than two the functions which can be obtained from $g(z ; n, \chi)$ by translation along the trajectories of $Q(w ; n, \chi) d w^{2}$ are a one parameter family $G(z ; n, \chi, \mu)$ where

$$
G(z ; n, \chi, \mu)=z\left(1+\frac{1}{2} n \mu e^{n \chi i / 2} z^{-n / 2}+e^{n \times i} z^{-n}\right)^{2 / n},
$$


the root is chosen so that the expansion of $G(z ; n, \chi, \mu)$ about the point at infinity begins

$$
z+\mu e^{n \chi^{i / 2} z^{-n / 2+1}}+\left(\frac{2}{n}-\frac{n-2}{4} \mu^{2}\right) z^{-n+1}+\cdots
$$

and $\mu(-4 / n \leqq \mu \leqq 4 / n)$ denotes the (sensed) amount of translation in the $Q$ metric. The same formulae remain valid for $n=2$ if we restrict ourselves to functions not assuming the value zero for $|z|>1$.

This is proved in a straightforward manner.

3. TheOREM 1. Let $f(z) \in \Sigma$ and have the expansion about the point at infinity

$$
f(z)=z+\sum_{j=0}^{\infty} \frac{c_{j}}{z^{j}}
$$

where $c_{j}=0$ for $j \leqq(n-1) / 2, n$ an integer $\geqq 0$. Further for $n=0$ let $f(z)$ not assume the value zero for $|z|>1$. Then

$$
\left|c_{n}\right| \leqq \frac{2}{n+1}
$$

Equality can occur in (2) only for the functions $g(z ; n+1, \chi), \chi$ real.

Let $\Phi(w)$ denote the inverse of $g(z ; n+1, \chi)$ defined in $\Delta(n+1, \chi)$. Then we apply the General Coefficient Theorem in its extended form [5, Theorem 1] with $\Re$ the $w$-sphere, $Q(z) d z^{2}$ being $Q(w ; n+1, \chi) d w^{2}$, the admissible domain $\Delta(n+1, \chi)$ and the admissible function $f(\Phi(w))$.

The latter function has the expansion about the point at infinity

$$
w+\sum_{j=0}^{\infty} \frac{d_{j}}{w^{j}}
$$

where $d_{j}=0$ for $j \leqq(n-1) / 2$ and $d_{n}=c_{n}-2 e^{(n+1) x^{i}} /(n+1)$. The quadratic differential has a pole $P_{1}$ of order $n+3$ at the point at infinity. We have $m_{1}=n+3, k_{1}=[n / 2]$. The corresponding coefficients are

$$
\alpha^{(1)}=e^{-(n+1) x^{i}}, \quad a_{n}^{(1)}=c_{n}-\frac{2}{n+1} e^{(n+1) x^{i}} .
$$

The fundamental inequality $[5,(6)]$ then gives

$$
\Re\left\{e^{-(n+1) x i}\left[c_{n}-\frac{2}{n+1} e^{(n+1) x i}\right]\right\} \leqq 0
$$

or 


$$
\Re\left\{e^{-(n+1) x i} C_{n}\right\} \leqq \frac{2}{n+1} .
$$

For appropriate choice of the real quantity $\chi$ we have

$$
\Re\left\{e^{-(n+1) \times{ }^{i} c_{n}}\right\}=\left|c_{n}\right|
$$

so that inequality (2) follows. The equality statement follows at once from the general equality conditions in the General Coefficient Theorem.

THEOREM 2. Let $f(z) \in \Sigma$ and have the expansion (1) about the point at infinity where $c_{j}=0$ for $j<n, n$ a positive integer. Then for real $\psi$

(3) $\Re\left\{-e^{-2(n+1) \psi i} c_{2 n+1}+\delta e^{-(n+1) \psi i} c_{n}-\frac{1}{2} n e^{-2(n+1) \psi i} c_{n}^{2}\right\}$

$$
\leqq \frac{3}{8(n+1)} \delta^{2}-\frac{1}{4(n+1)} \delta^{2} \log (\delta / 4)+\frac{1}{n+1}, \quad 0<\delta \leqq 4,
$$

(4) $\Re\left\{-e^{-2(n+1) \psi i} c_{2 n+1}-\frac{1}{2} n e^{-2(n+1) \psi i} c_{n}^{2}\right\} \leqq \frac{1}{n+1}$,

$$
\delta=0 \text {. }
$$

For $0 \leqq \delta<4$ equality occurs in (3) or (4) only for the functions $F(z ; n+1, \delta, \psi, \mu)$. For $\delta=4$ equality occurs in (3) only for the functions $f(z ; n+1,4, \psi)$. For $n=0$, (4) remains true without further restriction, but for (3) we must require that $f(z)$ does not take the value zero for $|z|>1$. The equality statement for (3) is unchanged, but for (4) equality can occur for any function $f(z ; 1,0, \psi)+k, k a$ constant.

Let $\Psi(w)$ denote the inverse of $f(z ; n+1, \delta, \psi)$ defined in $\Delta(n+1, \delta, \psi)$. Then we apply the General Coefficient Theorem (again in its extended form) with $\Re$ the $w$-sphere, $Q(z) d z^{2}$ being $Q(w ; n+1, \delta, \psi) d w^{2}$, the admissible domain $\Delta(n+1, \delta, \psi)$ and the admissible function $f(\Psi(w))$.

The latter function has the expansion about the point at infinity

$$
\begin{gathered}
w+\sum_{j=0}^{\infty} \frac{d_{j}}{w^{j}} \quad \text { where } d_{j}=0 \text { for } j<n, \\
d_{n}=c_{n}-\frac{1}{2(n+1)} \delta[1-\log (\delta / 4)] e^{(n+1) \psi i},
\end{gathered}
$$

and

$$
\begin{aligned}
d_{2 n+1}= & c_{2 n+1}+c_{n}\left\{\frac{n}{2(n+1)} \delta[1-\log (\delta / 4)] e^{(n+1) \psi i}\right\}-\left[\frac{2 n+1}{8(n+1)^{2}} \delta^{2}\right. \\
& \left.-\frac{2 n+1}{4(n+1)^{2}} \delta^{2} \log (\delta / 4)+\frac{n}{8(n+1)^{2}} \delta^{2}[\log (\delta / 4)]^{2}-\frac{1}{n+1}\right] e^{2(n+1) \psi i} .
\end{aligned}
$$


The quadratic differential has a pole $P_{1}$ of order $2 n+4$ at the point at infinity. We have $m_{1}=2 n+4, k_{1}=n$. The corresponding coefficients are

$$
\begin{aligned}
\alpha^{(1)} & =-e^{-2(n+1) \psi i}, \\
\epsilon_{1} & =1, \\
a_{k_{1}}^{(1)} & =d_{n}, \\
a_{m_{1}-3}^{(1)} & =d_{2 n+1}, \\
\beta_{k_{1}+1}^{(1)} & =-\delta e^{(n+1) \psi i} .
\end{aligned}
$$

The fundamental inequality then gives

$$
\begin{aligned}
& \Re\left\{-e^{-2(n+1) \psi i}\left[c_{2 n+1}+c_{n}\left(\frac{n}{2(n+1)} \delta(1-\log (\delta / 4)) e^{(n+1) \psi i}\right)\right.\right. \\
& -\left(\frac{2 n+1}{8(n+1)^{2}} \delta^{2}-\frac{2 n+1}{4(n+1)^{2}} \delta^{2} \log (\delta / 4)\right. \\
& \left.\quad+\frac{n}{8(n+1)^{2}} \delta^{2}(\log (\delta / 4))^{2}-\frac{1}{n+1}\right) e^{2(n+1) \psi i} \\
& \quad+\frac{1}{2} n\left(c_{n}-\frac{1}{2(n+1)} \delta(1-\log (\delta / 4)) e^{(n+1) \psi i}\right)^{2} \\
& \left.-\delta e^{(n+1) \psi i}\left(c_{n}-\frac{1}{2(n+1)} \delta(1-\log (\delta / 4)) e^{(n+1) \psi i}\right]\right\} \leqq 0
\end{aligned}
$$

or

$$
\begin{aligned}
\Re\left\{-e^{-2(n+1) \psi i} c_{2 n+1}+\delta e^{-(n+1) \psi i}\right. & \left.c_{n}-\frac{1}{2} n e^{-2(n+1) \psi i} c_{n}^{2}\right\} \\
& \leqq \frac{3}{8(n+1)} \delta^{2}-\frac{1}{4(n+1)} \delta^{2} \log (\delta / 4)+\frac{1}{n+1},
\end{aligned}
$$

proving inequality (3). Inequality (4) is proved by a similar but simpler argument. The equality statements follow from the standard equality conditions in the General Coefficient Theorem.

Corollary 1. Let $f(z) \in \Sigma$ and have the expansion (1) about the point at infinity where $c_{j}=0$ for $j<n, n$ a non-negative integer. Further for $n=0$ let $f(z)$ not assume the value zero for $|z|>1$. Then for $\alpha+n / 2<0$

$$
\left|c_{2 n+1}-\alpha c_{n}^{2}\right| \leqq \frac{1-n-4 \alpha}{(n+1)^{2}} .
$$

Equality occurs in (5) only for the functions $F(z ; n+1,0, \psi, \pm 2 /(n+1)), \psi$ real. For $\alpha+n / 2=0$ 


$$
\left|c_{2 n+1}+\frac{1}{2} n c_{n}^{2}\right| \leqq \frac{1}{n+1} .
$$

Equality occurs in (6) only for the functions $F(z ; n+1,0, \psi, \mu), \psi$ real, $-2 /(n+1)$ $\leqq \mu \leqq 2 /(n+1)$. For $-n / 2<\alpha<n / 2+1$

$$
\left|c_{2 n+1}-\alpha c_{n}^{2}\right| \leqq(n+1)^{-1}\left[1+2 \exp \left(-2 \frac{n-2 \alpha+2}{n+2 \alpha}\right)\right] .
$$

Equality occurs in (7) only for the functions

$$
f\left(z ; n+1,4 \exp \left(-\frac{n-2 \alpha+2}{n+2 \alpha}\right), \psi\right),
$$

$\psi$ real. For $\alpha \geqq n / 2+1$

$$
\left|c_{2 n+1}-\alpha c_{n}^{2}\right| \leqq \frac{4 \alpha+n-1}{(n+1)^{2}} .
$$

Equality occurs in (8) only for the functions $f(z ; n+1,4, \psi), \psi$ real.

We take the first inequality (4) and choose $\psi$ so that

$$
-\Re\left\{e^{-2(n+1) \psi i}\left(c_{2 n+1}+\frac{1}{2} n c_{n}^{2}\right)\right\}=\left|c_{2 n+1}+\frac{1}{2} n c_{n}^{2}\right|
$$

from which inequality (6) is immediate. Likewise the equality statement follows from Theorem 2. Now for $\alpha+n / 2<0$ we have

$$
\begin{aligned}
\left|c_{2 n+1}-\alpha c_{n}^{2}\right| & \leqq\left|c_{2 n+1}+\frac{1}{2} n c_{n}^{2}\right|-\left(\alpha+\frac{1}{2} n\right)\left|c_{n}^{2}\right| \\
& \leqq \frac{1}{n+1}-\left(\alpha+\frac{1}{2} n\right) \frac{4}{(n+1)^{2}} \\
& \leqq \frac{1-n-4 \alpha}{(n+1)^{2}} .
\end{aligned}
$$

Equality is possible only for those functions $F(z ; n+1,0, \psi, \mu)$ with $\left|c_{n+1}\right|$ $=2 /(n+1)$, that is $F(z ; n+1,0, \psi, \pm 2 /(n+1))$.

Now let $\alpha+n / 2>0$. Then by Theorem 2

$$
\Re\left\{-e^{-2(n+1) \psi i}\left(c_{2 n+1}-\alpha c_{n}^{2}\right)\right\} \leqq \frac{3}{8(n+1)} \delta^{2}-\frac{1}{4(n+1)} \delta^{2} \log (\delta / 4)+\frac{1}{n+1}
$$

$$
-\Re\left\{\delta e^{-(n+1) \psi i} c_{n}\right\}+\Re\left\{\left(\frac{1}{2} n+\alpha\right) e^{-2(n+1) \psi i} c_{n}^{2}\right\}, \quad 0<\delta \leqq 4 \text {, }
$$




$$
\begin{aligned}
\Re\left\{-e^{-2(n+1) \psi i}\left(c_{2 n+1}-\alpha c_{n}^{2}\right)\right\} \leqq \frac{1}{n+1}+\Re\left\{\left(\frac{1}{2} n+\alpha\right) e^{-2(n+1) \psi i} c_{n}^{2}\right\} & \\
& \delta=0 .
\end{aligned}
$$

We can choose $\psi$ real so that

$$
\Re\left\{-e^{-2(n+1) \psi i}\left(c_{2 n+1}-\alpha c_{n}^{2}\right)\right\}=\left|c_{2 n+1}-\alpha c_{n}^{2}\right|
$$

while

$$
\Re\left\{e^{-(n+1) \psi i} c_{n}\right\} \geqq 0 .
$$

Now in any case

$$
\Re\left\{e^{-2(n+1) \psi i} c_{n}^{2}\right\} \leqq\left[\Re\left\{e^{-(n+1) \psi i} c_{n}\right\}\right]^{2}
$$

with equality occurring only when

$$
\Im\left\{e^{-(n+1) \psi i} c_{n}\right\}=0 .
$$

Thus if

$$
\Re\left\{e^{-(n+1) \psi i} c_{n}\right\}=0,
$$

from (10) we obtain

$$
\left|c_{2 n+1}-\alpha c_{n}^{2}\right| \leqq \frac{1}{n+1} .
$$

Otherwise (by Theorem 1 ) we can find $\delta, 0<\delta \leqq 4$, so that

$$
\Re\left\{e^{-(n+1) \psi i} c_{n}\right\}=\frac{1}{2(n+1)} \delta[1-\log (\delta / 4)] .
$$

Then from inequalities (9) and (11) after a mechanical simplification we obtain

$$
\begin{aligned}
\left|c_{2 n+1}-\alpha c_{n}^{2}\right| \leqq \frac{1}{8}(n+1)^{-2}\left\{(2 \alpha-1) \delta^{2}-2(2 \alpha-1) \delta^{2} \log (\delta / 4)\right. \\
\left.+(n+2 \alpha) \delta^{2}[\log (\delta / 4)]^{2}+8(n+1)\right\} .
\end{aligned}
$$

Now we define

$$
\begin{array}{rrr}
\Lambda_{\alpha}(\delta) & =\frac{1}{8}(n+1)^{-2}\left\{(2 \alpha-1) \delta^{2}-2(2 \alpha-1) \delta^{2} \log (\delta / 4)\right. & \\
\left.+(n+2 \alpha) \delta^{2}[\log (\delta / 4)]^{2}+8(n+1)\right\}, & 0<\delta \leqq 4 \\
& =\frac{1}{n+\tau_{1} 1}, & \delta=0 .
\end{array}
$$


The function $\Lambda_{\alpha}(\delta)$ is continuous for $0 \leqq \delta \leqq 4$. Further we have

$$
\left|c_{2 n+1}-\alpha c_{n}^{2}\right| \leqq \max _{0 \leqq \delta} \Lambda_{\alpha}(\delta) .
$$

Now for $0<\delta \leqq 4$

$$
\frac{d \Lambda_{\alpha}(\delta)}{d \delta}=\frac{(n+2 \alpha) \delta \log (\delta / 4)}{4(n+1)^{2}}\left[\frac{n-2 \alpha+2}{n+2 \alpha}+\log (\delta / 4)\right] .
$$

Thus for $\alpha \geqq n / 2+1$ the function $\Lambda_{\alpha}(\delta)$ is a strictly increasing function on $[0,4]$, its maximum occurs for $\delta=4$ and

$$
\left|c_{2 n+1}-\alpha c_{n}^{2}\right| \leqq \frac{4 \alpha+n-1}{(n+1)^{2}} .
$$

Since we have used Theorem 2 , equality can occur here only for the functions $f(z ; n+1,4, \psi)$. Finally for $-n / 2<\alpha<n / 2+1$ the function $\Lambda_{\alpha}(\delta)$ increases from $\delta=0$ up to the point where

$$
\frac{n-2 \alpha+2}{n+2 \alpha}+\log (\delta / 4)=0
$$

that is

$$
\delta=4 \exp \left(-\frac{n-2 \alpha+2}{n+2 \alpha}\right),
$$

and then decreases. Thus the maximum of $\Lambda_{\alpha}(\delta)$ occurs for this value of $\delta$ and

$$
\left|c_{2 n+1}-\alpha c_{n}^{2}\right| \leqq(n+1)^{-1}\left[1+2 \exp \left(-2 \frac{n-2 \alpha+2}{n+2 \alpha}\right)\right] .
$$

Since we have used Theorem 2 and inequality (11), equality can occur here only for the functions $f(z ; n+1,4 \exp (-(n-2 \alpha+2) /(n+2 \alpha)), \psi), \psi$ real.

Corollary 2. Let $f(z) \in \Sigma$ and have the expansion (1) about the point at infinity where $c_{j}=0$ for $j<n, n$ a positive integer. Then

$$
\left|c_{2 n+1}\right| \leqq(n+1)^{-1}\left[1+2 \exp \left(-2 \frac{n+2}{n}\right)\right] \text {. }
$$

Equality occurs in (12) only for the functions $f(z ; n+1,4 \exp (-2(n+2) / n), \psi)$, $\psi$ real.

This follows at once by specializing Corollary 1 to the case $\alpha=0$.

One can use Theorem 2 to solve other extremal problems-for example, to find the bounds for $\left|c_{2 n+1}\right|-\left|c_{n}\right|$ under the assumptions of Theorem 2 . 
4. Lemma 4. Let $f(z) \in M$ and have the expansion

$$
z+\sum_{j=2}^{\infty} A_{j} z^{i}
$$

about the origin where $A_{j}=0$ for $j<n, n$ an integer $\geqq 2$. Then $1 / f(1 / z) \in \Sigma$ and has the expansion (1) about the point at infinity where

$$
\begin{aligned}
c_{j} & =-A_{j+2}, & j \leqq 2 n-4, \\
c_{2 n-3} & =A_{n}^{2}-A_{2 n-1} . &
\end{aligned}
$$

If in addition $f(z) \in S$ then $1 / f(1 / z)$ does not take the value zero for $|z|>1$ and conversely.

This is immediate.

THEOREM 3. Let $n$ be an integer $\geqq 2$, let $f(z) \in S(n=2), f(z) \in M(n>2)$ and have the expansion (13) about the origin where $A_{j}=0$ for $j \leqq(n+1) / 2$. Then

$$
\left|A_{n}\right| \leqq \frac{2}{n-1} \text {. }
$$

Equality can occur in (14) only for the functions $1 / g(1 / z ; n-1, \chi)$, $\chi$ real.

This follows at once from Theorem 1 and Lemma 4. Prawitz [6] obtained this result in the more special case that $f(z) \in S$ without regard to the value of $n$. Had he proved it for $f(z) \in M, n>2$, he would have anticipated Schiffer and Golusin in obtaining the bound $\left|c_{2}\right| \leqq 2 / 3$ by about ten years.

THEOREM 4. Let $n$ be an integer $\geqq 2$, let $f(z) \in S(n=2), f(z) \in M(n>2)$ and have the expansion (13) about the origin where $A_{j}=0$ for $j<n$. Then for real $\psi$

$$
\begin{array}{ll}
\Re\left\{e^{-2(n-1) \psi i} A_{2 n-1}-\delta e^{-(n-1) \psi i} A_{n}-\frac{1}{2} n e^{-2(n-1) \psi i} A_{n}^{2}\right\} & \\
\leqq \frac{3}{8(n-1)} \delta^{2}-\frac{1}{4(n-1)} \delta^{2} \log (\delta / 4)+\frac{1}{n-1}, & 0<\delta \leqq 4, \\
\Re\left\{e^{-2(n-1) \psi i} A_{2 n-1}-\frac{1}{2} n e^{-2(n-1) \psi i} A_{n}^{2}\right\} \leqq \frac{1}{n-1}, & \delta=0 .
\end{array}
$$

For $0 \leqq \delta<4$ equality occurs in (15) or (16) only for the functions $1 / F(1 / z ; n-1, \delta, \psi, \mu)$. For $\delta=4$ equality occurs in (15) only for the functions $1 / f(1 / z ; n-1,4, \psi)$. For $n=2$, (16) remains true for $f(z) \in M$ but with the analogous revision of the equality statement to that in Theorem 2.

This follows at once from Theorem 2 and Lemma 4. 
Corollary 3. Let $n$ be an integer $\geqq 2$, let $f(z) \in S(n=2), f(z) \in M(n>2)$ and have the expansion (13) about the origin where $A_{j}=0$ for $j<n$. Then for $\beta>n / 2$

$$
\left|A_{2 n-1}-\beta A_{n}^{2}\right| \leqq \frac{4 \beta-n-1}{(n-1)^{2}} .
$$

Equality occurs in (17) only for the functions $1 / F(1 / z ; n-1,0, \psi, \pm 2 /(n-1))$, $\psi$ real. For $\beta=n / 2$

$$
\left|A_{2 n-1}-\frac{1}{2} n A_{n}^{2}\right| \leqq \frac{1}{n-1} .
$$

Equality occurs in (18) only for the functions $1 / F(1 / z ; n-1,0, \psi, \mu), \psi$ real, $-2 /(n-1) \leqq \mu \leqq 2 /(n-1)$. For $n / 2>\beta>1-n / 2$

$$
\left|A_{2 n-1}-\beta A_{n}^{2}\right| \leqq(n-1)^{-1}\left[1+2 \exp \left(-2 \frac{n+2 \beta-2}{n-2 \beta}\right)\right] .
$$

Equality occurs in (19) only for the functions

$$
1 / f\left(1 / z ; n-1,4 \exp \left(-\frac{n+2 \beta-2}{n-2 \beta}\right), \psi\right),
$$

$\psi$ real. For $\beta \leqq 1-n / 2$

$$
\left|A_{2 n-1}-\beta A_{n}^{2}\right| \leqq \frac{1+n-4 \beta}{(n-1)^{2}} .
$$

Equality occurs in (20) only for the functions $1 / f(1 / z ; n-1,4, \psi), \psi$ real.

This follows from Corollary 1 and Lemma 4.

Corollary 4. Let $n$ be an integer $\geqq 2$, let $f(z) \in S(n=2), f(z) \in M(n>2)$ and have the expansion (13) about the origin where $A_{j}=0$ for $j<n$. Then

$$
\left|A_{2 n-1}\right| \leqq(n-1)^{-1}\left[1+2 \exp \left(-2 \frac{n-2}{n}\right)\right] \text {. }
$$

Equality occurs in (21) only for the functions

$$
1 / f\left(1 / z ; n-1,4 \exp \left(-\frac{n-2}{n}\right), \psi\right),
$$

$\psi$ real.

This follows at once by specializing Corollary 3 to the case $\beta=0$.

It should be observed that the preceding results include as special cases all known precise bounds for the coefficients of functions in $S$ and $\Sigma$ apart 
from the bound $\left|A_{4}\right| \leqq 4$. Further many other extremal problems can be solved on the basis of them.

It is interesting to observe that if we take $n=3$ in Corollary 4 we obtain the result proved by Fekete and Szegö [1] under the assumptions that $f$ belonged to $S$ and was odd. Their proof made strong use of these assumptions, but it is seen that the validity of the result does not depend on them but only on the conditions $f(z) \in M, A_{2}=0$. A similar remark applies to Golusin's rather natural generalization of the Fekete-Szegö result to functions with higher degrees of symmetry [2].

\section{BIBLIOGRAPHY}

1. M. Fekete and G. Szegö, Eine Bemerkung über ungerade schlichte Funktionen, J. London Math. Soc. vol. 8 (1933) pp. 85-89.

2. G. M. Golusin, Some problems in the theory of univalent functions, Trudy Mat. Inst. Steklov. no. 27 (1949) (Russian).

3. James A. Jenkins, Univalent functions and conformal mapping, Berlin-Göttingen-Heidelberg, Springer-Verlag, 1958.

4. - On certain coefficients of univalent functions, "Analytic functions," Princeton, Princeton University Press, 1960, pp. 159-194.

5. - An extension of the general coefficient theorem, Trans. Amer. Math. Soc. vol. 95 (1960) pp. 387-407.

6. H. Prawitz, Über Mittelwerte analytischer Funktionen, Ark. Mat. vol. 20A no. 6 (1927) pp. 1-12.

Institute for Advanced Study,

Princeton, New Jersey 